\title{
Effects of the illumination NA on EUV mask inspection with coherent diffraction imaging
}

\author{
Ricarda Nebling, Hyun-su Kim, Uldis Locans, Atoosa Dejkameh, Yasin Ekinci, and Iacopo \\ Mochi
}

Paul Scherrer Institut, 5232 Villigen PSI, Switzerland

\begin{abstract}
RESCAN is a coherent diffraction imaging based APMI microscope prototype. A complex image of the EUV reticle is reconstructed from diffraction patterns collected on a CCD detector. With the next upgrade of the tool, the resolution will be enhanced from the current $34 \mathrm{~nm}$ down to $20 \mathrm{~nm}$ on mask. Also the illumination NA value will change from the current range of 0.002 to 0.02 to a value of 0.035 . Here, we study how a change of the illumination NA affects the EUV mask inspection in simulation. We observe a better image quality, lower object error and higher defect sensitivity with increasing illumination NA.
\end{abstract}

Keywords: EUV lithography, actinic patterned mask inspection, coherent diffraction imaging, ptychography, illumination NA

\section{INTRODUCTION}

RESCAN (reflective-mode EUV mask scanning lensless microscope) is an APMI microscope prototype developed at the Paul Scherrer Institut. It is based on a coherent diffraction imaging ${ }^{1}$ technique called ptychography, ${ }^{2,3}$ where the reticle is scanned with spatially-confined, coherent EUV illumination in overlapping positions. On a CCD detector in the far-field, the diffraction patterns for each scan position are collected. The (complex) image of the reticle is reconstructed from the intensity data by using a phase-retrieval algorithm.

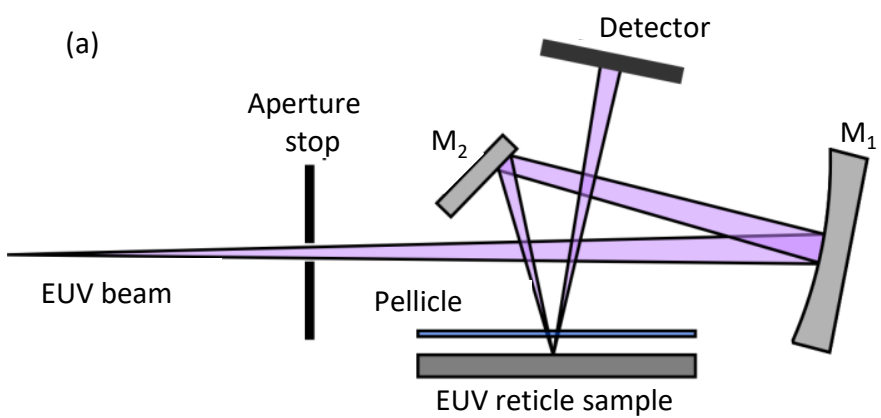

(b)

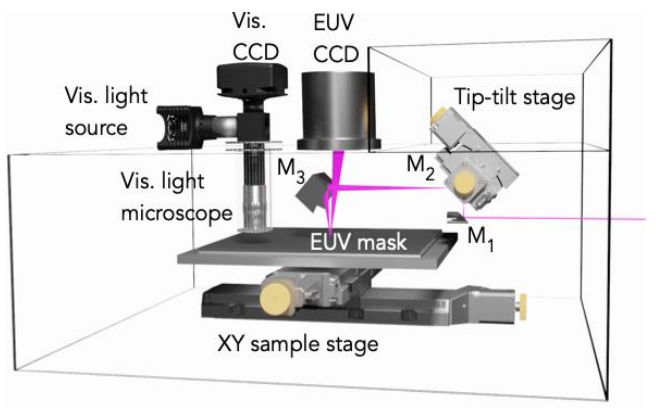

Figure 1. (a) Schematic of the current RESCAN setup. (b) Rendering of the upgraded RESCAN setup, with a Fourier synthesis illuminator.

The current RESCAN tool (the optical layout is depicted in fig. 1 (a)), has a resolution of $34 \mathrm{~nm}$ on mask. The EUV beam is focused onto the sample with an angle of incidence of $6^{\circ}$ and the illumination numerical aperture (NA) value ranges from 0.002 to $0.02 .{ }^{4}$ An upgrade of the tool is under construction: an image of the new design is shown in fig. 1 (b). The two mirror system will be replaced by a Fourier synthesis illuminator, which will

Further author information:

E-mail: ricarda.nebling@psi.ch

Extreme Ultraviolet Lithography 2020, edited by Patrick P. Naulleau, Paolo A. Gargini, Toshiro Itani, Kurt G. Ronse, Proc. of SPIE Vol. 11517, 115170W · (C) 2020 SPIE · CCC code: 0277-786X/20/\$21 · doi: 10.1117/12.2573181 
enhance the resolution on mask down to $20 \mathrm{~nm} .{ }^{5}$ A visible light microscope will facilitate the navigation on the sample. Furthermore, the illumination NA changes to a larger value of 0.035 .

In this paper, we study in simulation how a change in the illumination NA influences the EUV mask inspection with coherent diffraction imaging.

\section{ILLUMINATION NA SIMULATION}

In this study, we are performing defect inspection on simulated data sets with illumination NA values ranging from $\mathrm{NA}_{\text {illum }}=0.005$ to $\mathrm{NA}_{\text {illum }}=0.050$. The lowest $\mathrm{NA}$ value corresponds to the typical illumination NA of the current RESCAN setup, the range is chosen to cover the illumination NA for the RESCAN upgrade $\left(\mathrm{NA}_{\text {illum }}=0.035\right)$. As our current RESCAN tool is limited to a maximum illumination NA of 0.02, we study the influence of the illumination NA on EUV mask inspection in simulation only.

The EUV mask sample is generated from a mask design file with programmed absorber defects, varying in size from $200 \mathrm{~nm}$ to $20 \mathrm{~nm}$. We assume the sample to be binary, and add a phase shift to the absorber layer, that corresponds to $70 \mathrm{~nm}$ TaBN under $6^{\circ}$ angle of incidence at a wavelength of $13.5 \mathrm{~nm}$. The complex illumination function (also called probe) is simulated matching the characteristics of the current RESCAN setup (fig. 1 (a)). It is generated as an image of the beam shaping aperture on the reticle, with a wavelength of $13.5 \mathrm{~nm}$ (EUV). The illumination NA is determined by the aperture stop diameter and by its distance from the sample. The EUV reticle is moved through focus to maintain a constant probe diameter of $10 \mu \mathrm{m}$ on object for all data sets. Images of the probe magnitudes are shown in fig. 2.
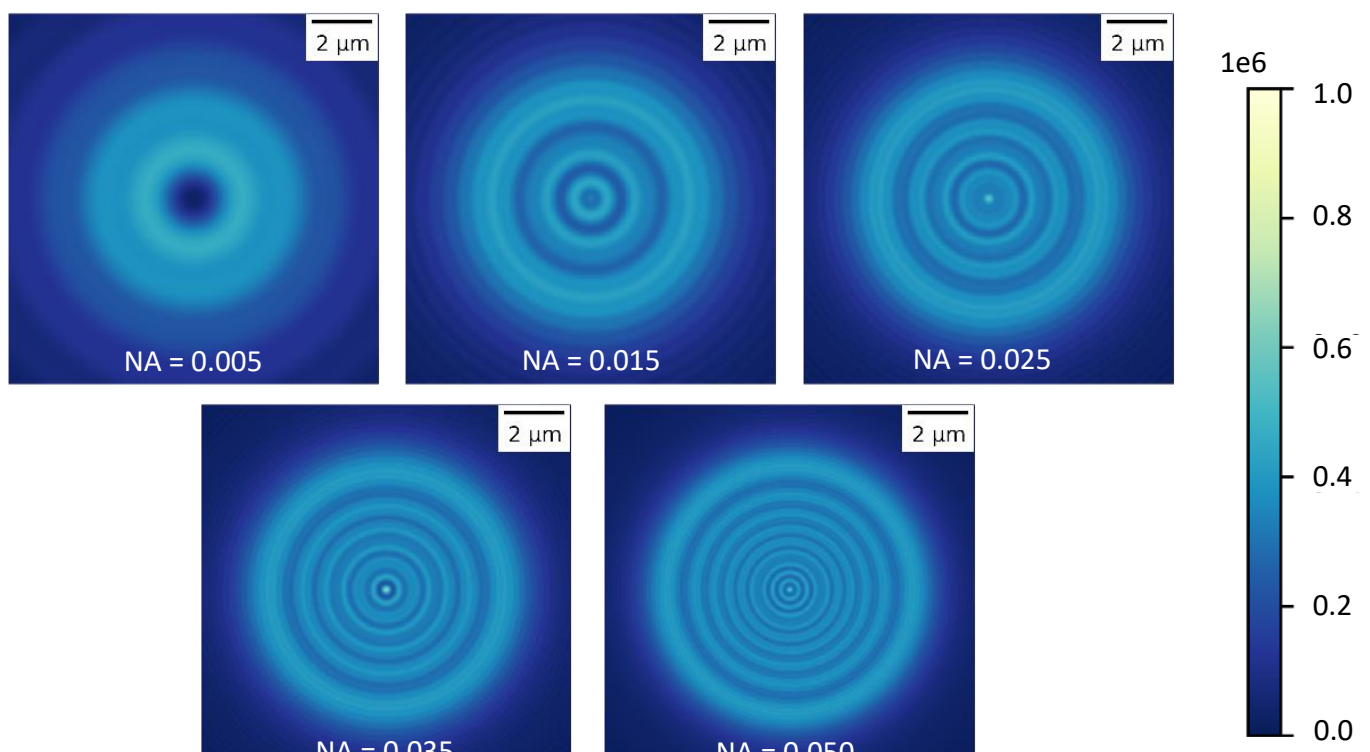

Figure 2. Simulated illumination magnitudes without noise on the object plane. From left to right and top to bottom increasing illumination NA.

To generate the diffraction patterns, the probe function is multiplied with the object region, that corresponds to the current scan position. The product corresponds to the exit wave and is propagated to the detector in the far-field, using a Fourier transform and an appropriate phase term. ${ }^{6}$ The squared absolute value of the propagated exit wave gives the noise-free diffraction pattern. To get the full ptychographic data set, this procedure is repeated for all scan positions. The average detector count per data set is scaled according to real RESCAN data. We add Poisson noise to each diffraction pattern. The sample is scanned in a circular pattern with a step size of $1 \mu \mathrm{m}$ to avoid regular grid pathology. ${ }^{7}$ 


\section{EUV RETICLE IMAGE RECONSTRUCTION}

We reconstruct a (complex) image of the EUV mask for each illumination function using the difference map algorithm. ${ }^{7}$ The algorithm is run for 250 iterations, while updating the probe function from the 100th iteration on. The initial object is generated following a random uniform distribution of values between zero and one, the initial probes are the complex illumination functions as simulated.

To compare the reconstructions quantitatively, we introduced the object error ${ }^{8}$

$$
E_{\text {obj }}=\frac{\sum_{\mathbf{r}} \sum_{i, j}\left\|O_{i j}^{r e f}|-| \hat{O}_{i j}\right\|^{2}}{\sum_{\mathbf{r}} \sum_{i, j} 1} .
$$

The absolute value of the reconstructed object $\hat{O}$ is compared at each iteration to a reference object $O^{\text {ref }}$ from the sample layout. The error is normalized to the total number of pixels. The object error for all NAs is shown in fig. 3. After the probe update starts, at the 100th iteration, the error is decaying fast to a steady level for all the illumination NAs. Fluctuations around that level, best visible for the lower NA curves, are inherent to the difference map algorithm, that typically reaches a steady state close to the optimal solution. ${ }^{7}$ To find the optimal image reconstruction, one can average the solutions of the last few iterations, ${ }^{7}$ or run for more iterations with another algorithm (for example from the PIE-family ${ }^{9}$ ) that is known to converge more likely to the global minimum. We observe a larger object error for the two smallest NAs, and a smaller object error for the largest three illumination NAs. The lowest error is observed for the largest NA $\left(\mathrm{NA}_{\text {illum }}=0.050\right)$. From fig. 3, we observe a trend for a lower object error and hence a more accurate reconstruction of the sample, with larger illumination NA.

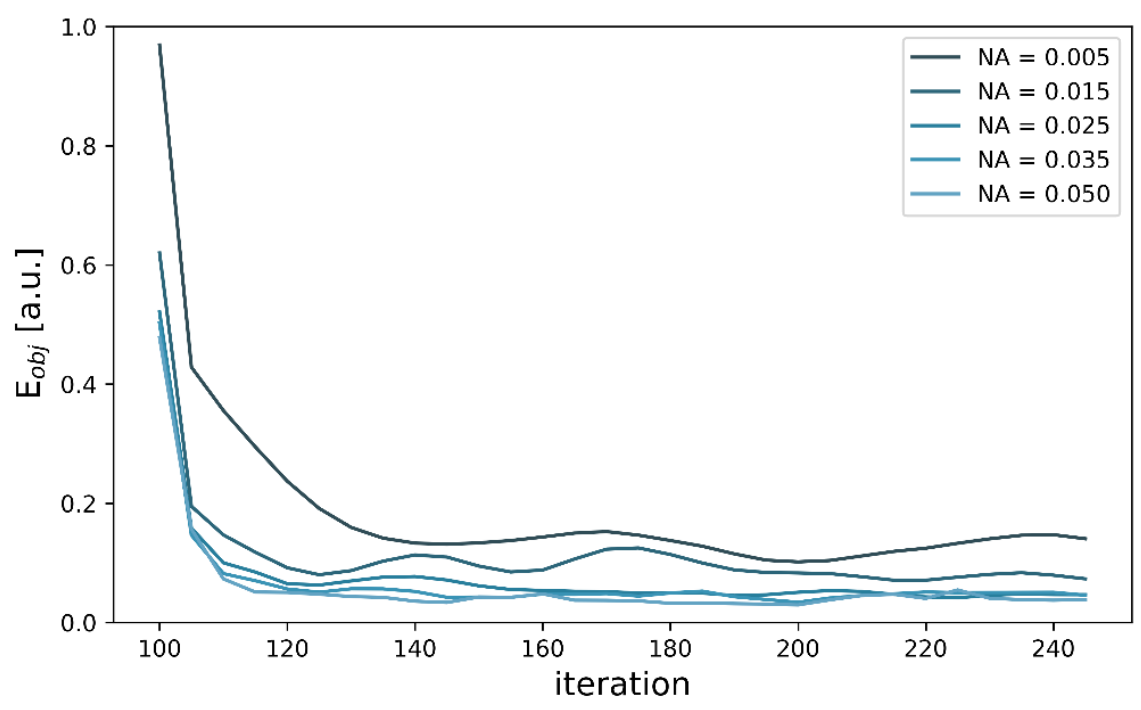

Figure 3. Object error for the illumination NAs.

\section{DEFECT DETECTION}

The simulated EUV mask contains an area with planned defects ranging in size from $200 \mathrm{~nm}$ to $20 \mathrm{~nm}$. A schematic of the region is shown in fig. 4 (c). Each of the nine crosses contains line intrusions and extrusions, one corner defect and one pin-dot defect with respective size. The critical dimension of the sample is $200 \mathrm{~nm}$. To detect the defects, we perform a die-to-database comparison for each of the reconstructed mask images. ${ }^{4} \mathrm{~A}$ die with defects, depicted in fig. 4 (a), is compared to the defect free reference shown in 4 (b). 
(a)

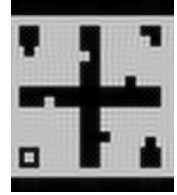

(b)

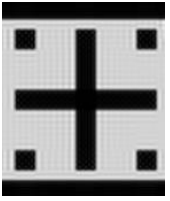

(c)
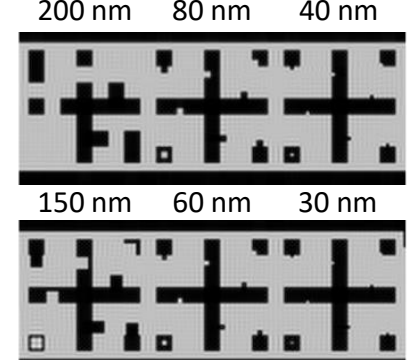

$100 \mathrm{~nm} \quad 50 \mathrm{~nm} \quad 20 \mathrm{~nm}$

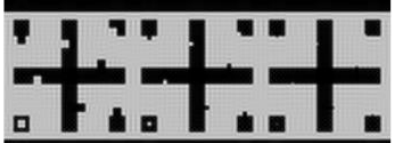

$-750 \mathrm{~nm}$

(e)

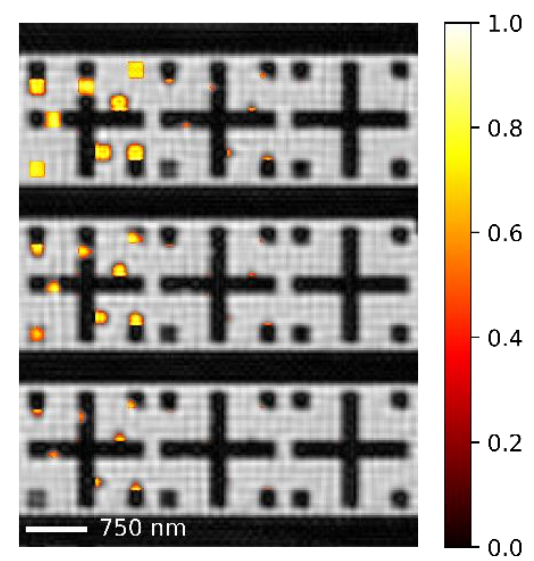

(d)

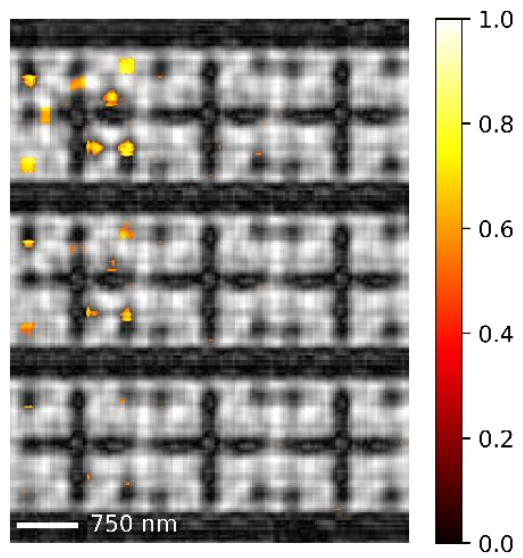

(f)

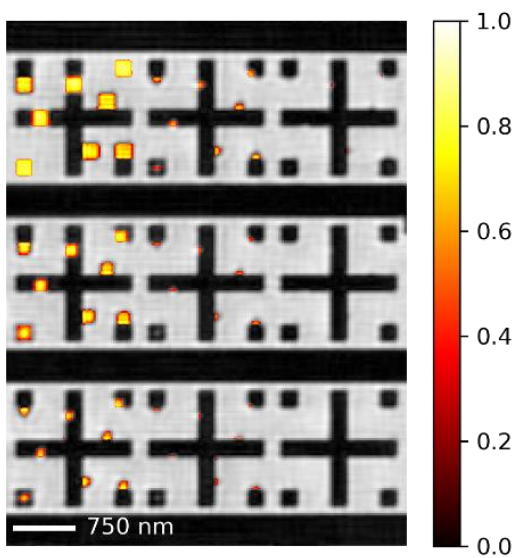

Figure 4. Defect maps for three different illumination NAs. (a) Schematic of a sample area with defects and (b) the respective reference from the database. (c) Layout of the sample design with defect sizes ranging from $200 \mathrm{~nm}$ to $20 \mathrm{~nm}$. (d) Shows the defect map overlaid on the reconstructed image magnitude for an illumination NA of 0.005 , (e) for an illumination NA of 0.025 , and (f) from an illumination NA of 0.050 , from a die-to-database comparison.

In fig. 4 (d), (e), and (f), the defect maps for three different illumination NA values $\left(\mathrm{NA}_{\text {illum }}=0.005\right.$, $\mathrm{NA}_{\text {illum }}=0.025$, and $\left.\mathrm{NA}_{\text {illum }}=0.050\right)$ are shown overlaid with the reconstructed EUV mask image magnitudes. From a first look, we observe a better image quality for the larger illumination NA reconstructions. The features are better resolved and the pattern is well visible for the largest illumination NA in fig. 4 (f). Considering all three defect maps, we see that more defects are detected down to smaller defect sizes for a larger illumination NA. It is important to note that this is a simulation study. For real RESCAN data we demonstrated defect detection down to 50 by $50 \mathrm{~nm}^{2}$ with an illumination NA of $0.002 .{ }^{4}$

To look more closely on the influence of the illumination NA on EUV mask inspection with coherent diffraction imaging, we listed the detected defect signals for different illumination NA and defect size in a table for each defect type. The tables for line intrusions (left) and extrusions (right) are shown in fig. 5. For both line defect types, smaller defects are detected with increasing illumination NA. Please note, that the defect signals in the table are not corrected for false positives.

In fig. 6 the same tables for corner and pin-dot defects are shown. For all defect types, we observe a higher defect sensitivity with larger illumination NA. 


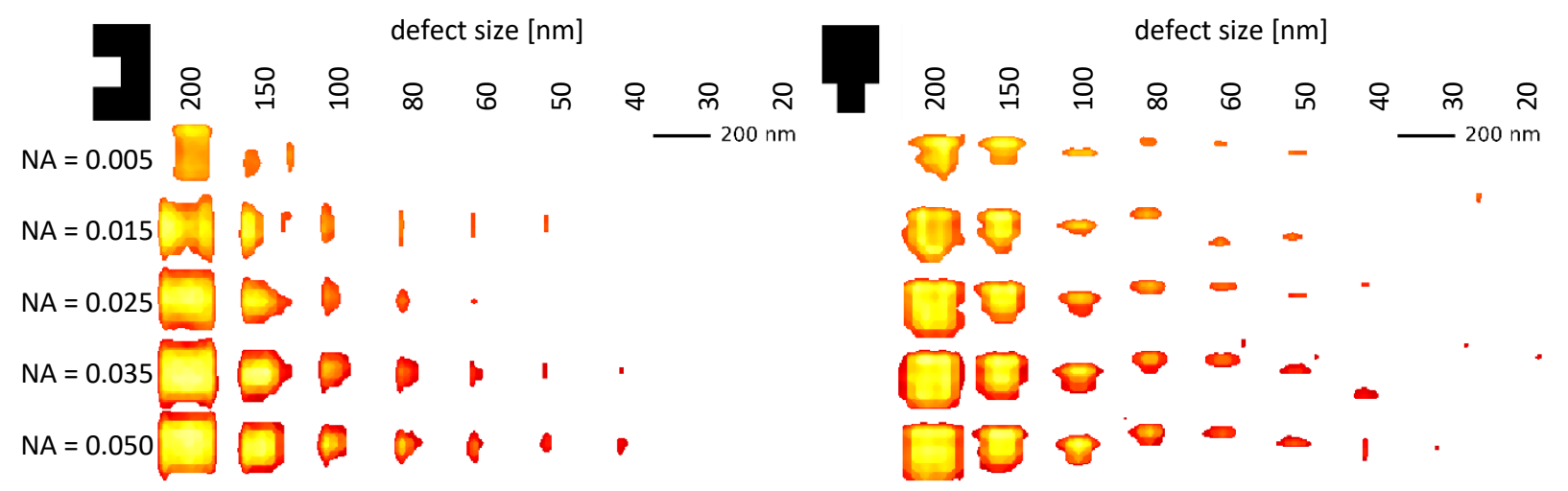

Figure 5. Table containing the detected defect signals for the different illumination NAs with changing defect size. On the left, a table with line intrusion defects is shown, while on the right, a table with line extrusion defects is shown.

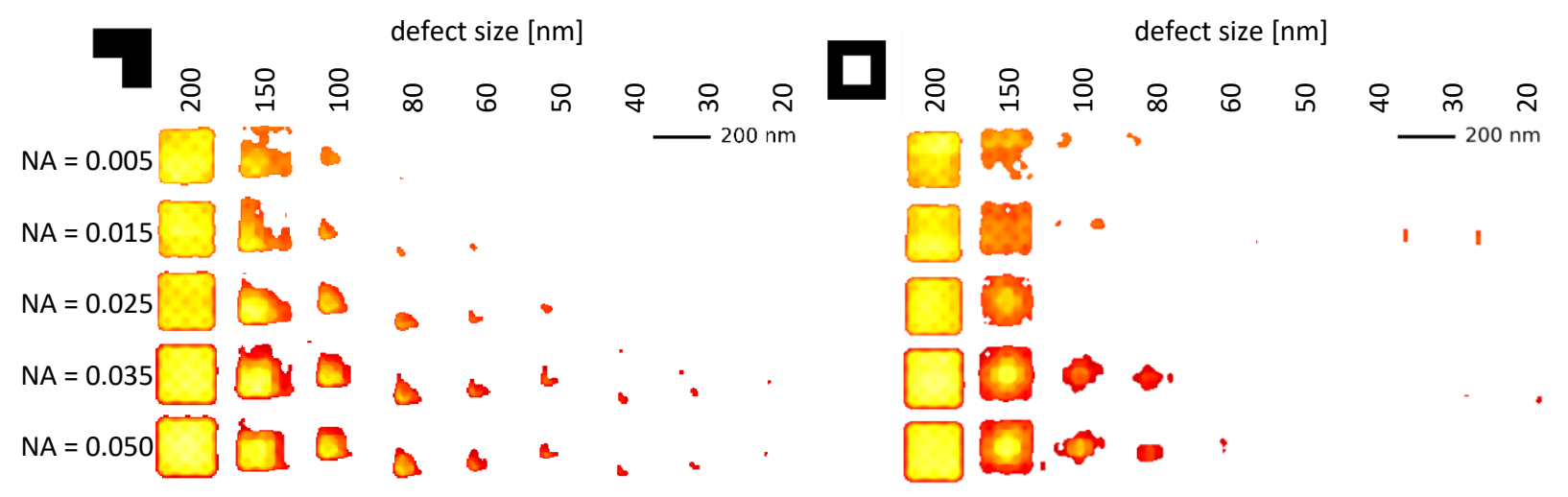

Figure 6. Left: Table showing the corner defect signals for different NAs and changing defect size. On the right, the same table with pin-dot defects is shown.

\section{CONCLUSIONS AND OUTLOOK}

In this paper, we studied the effects of the illumination NA on EUV reticle inspection with coherent diffraction imaging. We first simulated ptychographic data sets for several illumination functions with illumination NA values ranging from $\mathrm{NA}_{\text {illum }}=0.005$ to $\mathrm{NA}_{\text {illum }}=0.050$, and reconstructed the (complex) EUV mask image using the difference map algorithm.

We observed that larger illumination NA values yield lower object errors. The reconstructed image quality is better for a larger illumination NA and the pattern is better resolved. We furthermore observe a higher defect sensitivity with larger illumination NA values. More and smaller defects are detected in a die-to-database comparison for the large illumination NA data sets.

We expect that with the RESCAN upgrade to a Fourier synthesis illuminator and an increased illumination $\mathrm{NA}$, we will enhance the resolution to $20 \mathrm{~nm}$ on mask and get a higher defect sensitivity.

\section{REFERENCES}

[1] Chapman, H. N. and Nugent, K. A., "Coherent lensless X-ray imaging," Nat. Photonics 4(12), 833-839 (2010).

[2] Hoppe, W., "Beugung im inhomogenen Primärstrahlwellenfeld. III. Amplituden- und Phasenbestimmung bei unperiodischen Objekten," Acta Crystallogr. Sect. A 25(4), 508-514 (1969).

[3] Rodenburg, J. M., "Ptychography and related diffractive imaging methods," Adv. Imaging Electron Phys. 150(07), 87-184 (2008). 
[4] Mochi, I., Fernandez, S., Nebling, R., Locans, U., Rajeev, R., Dejkameh, A., Kazazis, D., Tseng, L.-T., Danylyuk, S., Juschkin, L., and Ekinci, Y., "Quantitative characterization of absorber and phase defects on EUV reticles using coherent diffraction imaging," J. Micro/Nanolithography, MEMS, MOEMS 19(1), 014002 (2020).

[5] Mochi, I., Kim, H.-s., Locans, U., Dejkameh, A., Nebling, R., Kazazis, D., and Ekinci, Y., "Illumination control in lensless imaging for EUV mask inspection and review," in [Proc. SPIE 11323 Extrem. Ultrav. Lithogr. XI], (11323I) (2020).

[6] Voelz, D. G., [Computational Fourier Optics], SPIE Press, Bellingham, Washington USA, 3rd ed. (2011).

[7] Thibault, P., Dierolf, M., Bunk, O., Menzel, A., and Pfeiffer, F., "Probe retrieval in ptychographic coherent diffractive imaging," Ultramicroscopy 109(4), 338-343 (2009).

[8] Nebling, R., Mochi, I., Kazazis, D., Locans, U., Dejkameh, A., and Ekinci, Y., "EUV reticle inspection using phase retrieval algorithms: a performance comparison," in [Proc. SPIE 11147, Int. Conf. Extrem. Ultrav. Lithogr. 2019], (111470R) (2019).

[9] Maiden, A., Johnson, D., and Li, P., "Further improvements to the ptychographical iterative engine," Optica 4(7), $736-745$ (2017). 